\title{
Expertentreffen obstruktive Atemwegserkrankungen: Kardiovaskuläre Aspekte der COPD*
}

\section{Expert Meeting Chronic Obstructive Airway Disease - Cardiovascular Aspects of COPD}

Autoren

Institute
J. Lorenz ${ }^{1}$, R. Bals ${ }^{2}$, H. Magnussen ${ }^{3}$, M. Pfeifer ${ }^{4}$, W. Randerath ${ }^{5}$, G. Steinkamp ${ }^{6}$, C. Taube ${ }^{7}$, H. Teschler ${ }^{8}$, C. Vogelmeier ${ }^{9}$, T. Welte ${ }^{10}$, H. Worth ${ }^{11}$

Die Institutsangaben sind am Ende des Beitrags gelistet. eingereicht 26.7.2013

akzeptiert 30.7 .2013

\section{Bibliografie \\ DOI http://dx.doi.org/ \\ 10.1055/s-0033-1344642 \\ Online-Publikation: 2.9.2013 \\ Pneumologie 2013; 67: 663-675 \\ (c) Georg Thieme Verlag KG \\ Stuttgart · New York \\ ISSN 0934-8387}

\section{Korrespondenzadresse}

Prof. Dr. med. Joachim Lorenz

Klinik für Pneumologie, Internistische Intensivmedizin, Infektiologie und Schlafmedizin Klinikum Lüdenscheid Paulmannshöher Straße 14 58515 Lüdenscheid Joachim.lorenz@klinikumluedenscheid.de

\section{Zusammenfassung \\ $\nabla$}

Die hier vorgestellten, aktuellen Forschungsergebnisse zur COPD sind einer neuen Qualität in der klinischen Wissenschaft zu verdanken. Es sind vor allem zwei neue Datenquellen, die in den letzten Jahren erschlossen wurden: große populationsbasierte, retrospektive Beobachtungsstudien mit bis zu 5-stelligen Fallzahlen einerseits und sehr intensiv untersuchte, prospektive Kohorten andererseits. In einem der Beiträge dieses Artikels wird das neben der amerikanischen ECLIPSE-Kohorte in Deutschland entstehende COSYCONET vorgestellt. Hier kann sich der Leser einen Eindruck über den wissenschaftlichen Umgang mit einer prospektiven Kohorte verschaffen.

Die Flut neuer Daten hat auch dazu beigetragen, dass die Aussagen des internationalen GOLD-Komitees komplexer geworden sind. Klinisch wichtig sind der Verzicht auf das Ergebnis des Bronchospasmolysetests als Diagnosekriterium der COPD und die Verwendung von Symptomfragebögen neuerdings auch des CCQ-Scores - als neue Dimension in der Beschreibung der Erkrankungsschwere. Außerdem wird die Bedeutung schwerer akuter Exazerbationen für das weitere Schicksal der Patienten hervorgehoben. Die im GOLD-Update von 2011 erstmals vorgestellten COPD-Typen I bis IV waren zunächst lediglich eine Expertenmeinung. Daher sind die jetzt vorliegenden Daten über die prognostische Relevanz der Klassifikation dringend notwendig.

Das Hauptthema der Tagung waren die kardiovaskulären Aspekte der COPD. Es wird deutlich, dass für Patienten mit COPD - über die Komorbidität zweier häufiger chronischer Erkrankungen hinaus - kardiovaskuläre Ereignisse von besonderer Bedeutung sind. Umgekehrt ist die fortgeschrittene COPD ein wichtiger Risikofaktor bei Erkrankungen

\section{Abstract \\ $\nabla$}

This overview presents data that take advantage of a new step of insight into COPD. Large population-based retrospective studies and intensively investigated prospective cohorts are two important sources of knowledge that have been recently developed. One of the contributions introduces the German COSYCONET which is on its way shortly after the American ECLIPSE cohort.

The vast amount of new data has also contributed to some corrections of the recommendations of the international GOLD committee. Clinically important are the waiver of the reversibility test for the diagnosis of COPD, the inclusion of sympotom scores to evaluate quality of life and the estimation of exacerbations. The COPD types I through IV were originally the result of expert opinion, but their impact on prognosis has recently been evaluated empirically.

The top issues of the expert meeting were cardiovascular aspects of COPD. Besides the comorbidity of two significant chronic diseases, it became clear that cardiovascular events have an outstanding significance for COPD patients. Inversely, advanced COPD is an important risk factor in cardiac and vascular diseases. The mutual influence of both disease entities does not only affect the long term progression but also the outcome of acute events like myocardial infarction and COPD exacerbation.

The following contributions investigate the topic with regard to epidemiology, the biology of vessels, and especially with regard to acute COPD exacerbations and pharmakotherapy. Recent evidence enables a fresh view on the cardiovascular toxicity of COPD medication and on possible protective effects of cardiovascular drugs (i.e. statins and ß-receptor antagonists) for patients with COPD.

\footnotetext{
* Sponsor: Boehringer Ingelheim Pharma GmbH \& Co KG
} 
des Herzens und der Gefäße. Diese gegenseitige Beeinflussung gilt sowohl für den Langzeitverlauf als auch für akute Komplikationen wie die COPD-Exazerbation und den Myokardinfarkt, und sie macht sich in harten Endpunkten wie der Letalität bemerkbar. In den folgenden Beiträgen wird das Thema im Hinblick auf die Epidemiologie, die kardiovaskuläre Biologie und im Besonderen auf seine Bedeutung in der COPD-Exazerbation untersucht. Die kardiovaskuläre Toxizität der Pharmakotherapie der COPD wird ebenso neu bewertet wie mögliche protektive Effekte von Pharmaka mit kardiovaskulärer Indikation (Statine und Betarezeptorenblocker) bei Patienten mit COPD.

\section{Neue Evidenz zur COPD aus dem vergangenen Jahr \\ $\nabla$}

\section{Helmut Teschler, Essen}

\section{Neue epidemiologische Daten}

Aus allen 50 Bundesstaaten der USA wurden kürzlich Ergebnisse einer telefonischen Umfrage bei rund 500000 zufällig ausgewählten Bürgern publiziert [1]. Für die COPD betrug die Gesamtprävalenz 6,3\% entsprechend 15 Millionen erwachsenen Personen. Wie erwartet, nahm mit dem Alter die Prävalenz der Erkrankung zu. Die Zahl der Frauen mit COPD war in dieser Umfrage höher als die der Männer. In Haushalten mit niedrigem Einkommen war der Anteil von COPD-Patienten höher, ebenso bei geschiedenen oder verwitweten Personen. In Bundesstaaten mit rigorosen Konzepten gegen das Zigarettenrauchen zeigte sich ein geringerer Anteil von COPD-Patienten.

Eine neue Auswertung der NHANES-Kohorte berücksichtigte ab 1999 auch COPD und Komorbiditäten [2]. Gegenübergestellt wurden 995 Patienten mit COPD und knapp 15000 Personen ohne diese Erkrankung. Komorbiditäten mit höherer Prävalenz bei COPD waren u.a. Herzinsuffizienz, koronare Herzkrankheit, Osteoporose, Arthritis und Krebserkrankungen. Überraschend häufig wurde von Harninkontinenz berichtet, nämlich bei 35\% der COPD-Patienten und bei $27 \%$ der Kontrollpersonen. Angesichts der Tatsache, dass sich die Inkontinenz als relevantes klinisches Problem bei COPD erwies, sollte man bei der Anamneseerhebung insbesondere in fortgeschrittenen Stadien der COPD häufiger nach Harninkontinenz fragen.

\section{Biomarker}

Eine Auswertung der ECLIPSE-Studie verglich die Häufigkeit pathologischer Ergebnisse von Leukozyten, Hs-CRP, IL-6 und Fibrinogen bei 1419 COPD-Patienten, 257 Rauchern und 180 Nichtrauchern [3]. Die genannten Biomarker waren bei 20 bis $29 \%$ der COPD-Patienten pathologisch verändert. Zwar hatten Raucher zu 23\% erhöhte Leukozyten-Zahlen, die anderen Biomarker waren jedoch bei maximal 7\% der Raucher und Nichtraucher erhöht. Die Mortalität von COPD-Patienten mit zwei oder mehr pathologischen Biomarkern war signifikant höher, ebenso die jährliche Exazerbationsrate.

In einer dänischen COPD-Kohorte kamen bei leichter betroffenen Patienten bestimmte Komorbiditäten häufiger vor, wenn zwei oder drei und nicht nur ein Biomarker pathologisch verändert waren [4]. Dazu gehörten die koronare Herzerkrankung, Myokardinfarkt, Pneumonie und Lungenkrebs.

\section{Plaques in der $\mathrm{A}$. carotis}

In der Rotterdam-Studie wurde die Intima-Media-Dicke der A. carotis sonografisch bestimmt [5]. Überschritt sie den Grenzwert von 2,5 mm, wurde zusätzlich ein MRT durchgeführt. Bei den 88 Patienten mit COPD war die Prävalenz verdickter Karotiden-Wände höher als bei den 920 Kontrollpersonen (55\%), und sie stieg mit zunehmendem Schweregrad auf bis zu 90\% bei schwerer COPD an. Im MRT wurden bei COPD häufiger Plaques gesehen, wobei deren Lipid-Kern für das Vorhandensein von vulnerablen im Unterschied zu kalzifizierten Plaques sprach. Diese Daten weisen auf einen Zusammenhang zwischen pulmonaler Inflammation und kardiovaskulärer Erkrankung hin. Mögliche pathophysiologische Zusammenhänge wurden in einem begleitenden Editorial diskutiert [6].

\section{Verlauf nach Exazerbation und Pneumonie}

Eine klinisch wichtige Arbeit ging der Frage nach, wie sich der Krankheitsverlauf in den 12 Monaten nach schwerer Exazerbation entwickelt [7]. Ausgewertet wurden 73106 Patienten, die wegen der ersten schweren COPD-Exazerbation hospitalisiert werden mussten. Die mittlere Überlebenszeit nach Exazerbation berechnete sich auf 3,6 Jahre. Die Prognose war also vergleichbar mit der des Bronchialkarzinoms im Stadium II. Während einer Nachbeobachtungszeit von 10 Jahren wurden die Intervalle bis zur jeweils nächsten Exazerbation immer kürzer, und die Letalität nahm mit jeder Exazerbation zu. Eine therapeutische Konsequenz aus diesen Daten wäre, den Patienten so zu stabilisieren, dass das Intervall zur nächsten Exazerbation möglichst lang ist. Generell sollte man nach akuten Ereignissen eine bessere medizinische Nachbetreuung über viele Monate anstreben.

Wenn bei einer Exazerbation im Röntgenbild des Thorax ein Infiltrat zu sehen ist, der Patient also eine Pneumonie hat, ist seine Letalität sowohl in der Klinik als auch in den darauf folgenden 30 Tagen deutlich höher. Dies zeigte eine Auswertung von 920 Patienten [8]. Daraus folgt, dass bei jeder COPD-Exazerbation mit Hospitalisation ein Röntgenbild anzufertigen ist. Mehr achten sollte man auch auf venöse Thromboembolien, denn das Lungenembolierisiko ist bei COPD signifikant erhöht [9]. Hilfreiche Labormarker zur Abgrenzung einer Exazerbation der COPD von Komplikationen wie Herzinsuffizienz, Stauung und Lungenembolie sind Pro-BNP und Troponin T.

\section{Medikamentöse Therapie}

Der Phosphodiesterase-4-Hemmer Roflumilast hat bei Typ-2Diabetes einen günstigen Einfluss auf den Zuckerstoffwechsel [10]. Bei 205 neu diagnostizierten Patienten mit Diabetes Typ 2 wurde über 12 Wochen entweder Roflumilast oder Plazebo verabreicht. Der HbA1c war unter Roflumilast bereits nach 2 Wochen signifikant niedriger als in der Kontrollgruppe. Hier wäre eine Zulassungserweiterung auf COPD-Patienten mit Diabetes mellitus zu diskutieren, wenn dieser positive Effekt in weiteren Studien bestätigt werden kann.

Zu Tiotropium gibt es neue Ergebnisse aus der UPLIFT-Studie [11]. Patienten im Stadium GOLD II, die knapp die Hälfte aller 5993 randomisierten Personen ausmachte, zeigten sowohl vor als auch nach Bronchodilatation signifikant höhere mittlere FEV1-Werte als die Kontrollgruppe. Der Effekt war bei GOLD-IIPatienten etwas stärker als in den Gruppen GOLD III und IV. Die Reduktion der Exazerbationen betrug 20\% pro Jahr bei GOLD II und $12 \%$ bei GOLD III/IV. Diese Daten unterstützen die klinische Praxis, Tiotropium bereits ab dem GOLD-Stadium II einzusetzen. 
Das Institut für Qualität und Wirtschaftlichkeit im Gesundheitswesen, IQWIG, hat im Juni 2012 eine Nutzenbewertung für Tiotropium veröffentlicht. Gegenüber Plazebo wird der Nutzen hinsichtlich der Häufigkeit von Exazerbationen und der gesundheitsbezogenen Lebensqualität als belegt angesehen. Im Vergleich zu lang wirksamen Beta-Sympathomimetika besteht aus Sicht von IQWIG ein Zusatznutzen hinsichtlich der Häufigkeit von Exazerbationen und der damit verbundenen Hospitalisationen.

Im Vergleich zu Salmeterol und Formoterol war Tiotropium überlegen. Dagegen sehen die Leitlinien für die Erstlinien-Dauertherapie bisher den gleichrangigen Einsatz von Tiotropium und LABAs vor.

\section{Lungenvolumenreduktion}

Eine aktuelle Auswertung der NETT-Studie beschrieb die Veränderungen von Lungenfunktion, Atemmuskelkraft und Body Mass Index bei Patienten mit operativer Lungenvolumenreduktion im Vergleich zu konservativ und rehabilitativ behandelten Patienten [12]. Die Zunahme des BMI korrelierte mit der Abnahme des Residualvolumens durch die operative Volumenreduktion. Da die Abnahme des Residualvolumens eng mit der Verbesserung des Wirkungsgrads der Atempumpe zusammenhängt, führt die erfolgreiche Volumenreduktion wahrscheinlich zu einer Verminderung des Energiebedarfs für die Atmung und ermöglicht so die beschriebene Gewichtszunahme.

\section{GOLD-Update 2011 und seine Implikationen}

$\nabla$

\section{Claus Vogelmeier, Marburg}

Im Februar 2013 wurde von GOLD, einer Organisation, der über 130 Nationen angehören, eine überarbeitete Fassung der Empfehlungen von 2011 im Internet publiziert (www.GOLDcopd.org) [13]. Darunter sind viele Schwellenländer und Staaten der Dritten Welt. Insofern kann die Ausrichtung nicht allein auf die westliche Welt zentriert sein. Im Folgenden werden die Änderungen gegenüber den GOLD-Leitlinien von 2009 und 2011 zusammengefasst dargestellt.

\section{Definition der COPD}

Die GOLD-Expertengruppe hat sich bei der erneuten Überarbeitung dafür entschieden, die ab 2013 gültige Version nicht mehr als Leitlinie zu bezeichnen, sondern als Positionspapier.

Die aktuelle Definition der Erkrankung hat - wie bisher - den Charakter einer Beschreibung:

Chronic Obstructive Pulmonary Disease (COPD), a common preventable and treatable disease, is characterized by persistent airflow limitation that is usually progressive and associated with an enhanced chronic inflammatory response in the airways and the lung to noxious particles or gases. Exacerbations and comorbidities contribute to the overall severity in individual patients.

Exazerbationen werden als neuer Bestandteil der Definition aufgeführt, ebenso wie die chronische Inflammation und die Ursachen der Erkrankung. Als Fortschritt ist zu werten, dass die Erkrankung als „üblicherweise progredient“ beschrieben wird. Hintergrund dafür sind Ergebnisse der ECLIPSE-Studie, die zeigten, dass viele Patienten langfristig einen stabilen Krankheitsverlauf hatten.
Spirometrie und Schweregrad-Klassifikation

Im Vereinigten Königreich werden mehr als 50\% der COPD-Diagnosen gestellt, ohne dass der Patient jemals mit einer Spirometrie untersucht worden ist. Die neuen GOLD-Kriterien fordern jetzt obligat (nicht mehr fakultativ) eine Spirometrie als Grundlage der Initialtherapie.

Die Klassifikation der Schweregrade blieb 2013 gegenüber der vorigen Version identisch. Die Diskussion, ob man besser die Ratio FEV1/FVC verwenden soll oder die untere Normgrenze der FEV1 bezogen auf Alter und Geschlecht, wurde in den letzten Jahren lebhaft geführt. Kliniker sprachen sich mehr für die Ratio aus, Physiologen für die untere Normgrenze. In einer Arbeit von Mannino wurde gezeigt, dass bei Personen über 60 Jahren die COPD überdiagnostiziert wird, wenn die untere Normgrenze verwendet wird [14]. Ein wichtiger Grund dafür liegt darin, dass nur für wenige Populationen verlässliche Sollwerte zur Verfügung stehen. Die Verwendung der Ratio gibt die pathologischen Verhältnisse gut wieder, wie eine neuere Arbeit von Mannino zeigt [15]. Patienten mit normaler Lungenfunktion (gemessen am unteren Normwert), gleichzeitig aber pathologischer Ratio, hatten eine doppelt so große Mortalität wie Patienten, bei denen beide Kriterien unauffällig waren.

\section{Reversibilitätstest}

Für die Testung der Reversibilität nach Gabe eines Bronchodilatators gibt es klare Durchführungsprotokolle. Die aktuelle Version von GOLD gibt allerdings keine Empfehlung hinsichtlich der Grenzwerte mehr. In der knapp 6000 Patienten umfassenden UPLIFT-Studie hatten nämlich rund 60\% der Patienten im GOLDStadium II sowie mehr als $20 \%$ im GOLD-Stadium IV ein positives Ergebnis des Reversibilitätstests, wenn die bisherigen Grenzwerte verwendet wurden [16]. Für die Abgrenzung gegenüber dem Asthma bronchiale ist das Ausmaß der FEV1-Reversibilität demnach nicht verwertbar.

Dass die Klassifikation der Lungenfunktions-Schweregrade sinnvoll ist und die Mortalität abbildet, wurde von verschiedenen Forschergruppen dokumentiert, beispielsweise von Mannino und Mitarbeitern [17]. Jedoch zeigten sich hinsichtlich des Ausmaßes der Dyspnoe und der Ergebnisse des 6-Minuten-Gehtests kaum Unterschiede in den verschiedenen Schweregrad-Gruppen, sondern eher breite und flach verlaufende Verteilungen [18, 19].

\section{Symptom-Fragebögen}

Die GOLD-Expertengruppe hat versucht, eine einfache Phänotypisierung mit leicht eruierbaren Parametern zu erarbeiten. Als Werkzeuge eignen sich der COPD-Assessment-Test (CAT) oder die mMRC-Atemnotskala, die in Deutschland kaum verwendet wird. In einem Vergleich zwischen dem St. George's Respiratory Questionnaire (SGRQ) als krankheitsspezifischem Lebensqualitätsfragebogen und der mMRC-Skala zeigten sich deutliche Überschneidungen in dem Sinne, dass in den einzelnen Stadien der Dyspnoe sehr unterschiedliche Grade von Lebensqualität vorkamen [20]. Eine vergleichende Untersuchung zwischen CAT und SGRQ zeigte eine gute Korrelation beider Punktewerte $(r=0,80)$ [21]. Der in knapp zwei Minuten auszufüllende CAT-Score könnte demnach im klinischen Alltag den umfangreichen Lebensqualitätsfragebogen ersetzen. Außerdem reagierte der CAT-Score auf Veränderungen und auf Interventionen. Bei Exazerbationen wurden nach vierzehntägiger Therapie signifikante Verbesserungen des CAT-Scores dokumentiert [22]. Ferner ließen sich Responder von Non-Respondern unterscheiden. In der ECLIPSE-Studie wa- 
ren die CAT-Scores bei Personen mit mehr als 2 Exazerbationen pro Jahr signifikant schlechter als bei Patienten mit selteneren Exazerbationen [23].

Als neues Element zur Beurteilung der Symptome wurde in die GOLD-Empfehlungen der CCQ-Fragebogen (Clinical COPD Questionnaire) aufgenommen [24]. Dieses für viele Sprachen validierte Instrument wird vorwiegend von Allgemeinärzten verwendet. Es beinhaltet 10 Fragen zu Dyspnoe, Aktivität, Husten, Sputum, Befürchtungen/Ängsten und Depression, und der Fragebogen kann in weniger als 2 min ausgefüllt werden. Ergebnisse unter 1 werden der leichten COPD-Kategorie zugeordnet.

\section{Neues Stratifizierungskonzept}

Die kombinierte Beurteilung der COPD erfolgt nunmehr mit Hilfe einer mehrdimensionalen Matrix. Sie beinhaltet die Kriterien spirometrisch gemessene Flusslimitation, Symptome (gemessen mit CAT, mMRC oder CCQ) und Exazerbationen. Inwiefern die gewählten Grenzwerte für ein erhöhtes Risiko optimal sind - beispielsweise ein mMRC-Wert von 2 oder mehr -, müssen zukünftige Studien zeigen. Aus den genannten Kriterien ergeben sich schließlich die vier Kategorien A bis D.

Als Hochrisikopatienten werden in der Version 2013 nicht nur Personen mit mindestens 2 Exazerbationen pro Jahr angesehen, sondern auch Patienten, die mindestens einmal im Jahr hospitalisiert wurden.

\section{Therapie der COPD}

Bei der Behandlung werden zunächst nicht-medikamentöse Maßnahmen wie Nikotinabstinenz, Impfungen und körperliche Aktivität hervorgehoben. Die pharmakologische Initialtherapie des Patienten richtet sich nach der Schweregradeinteilung. Substanzen der 1 . Wahl werden unterschieden von Medikamenten, die als erste bzw. als weitere Alternativen gelten. Die letztgenannte Kategorie bezieht sich vor allem auf Entwicklungsländer. Hier ist auch Theophyllin aufgeführt. In China wird derzeit eine große Studie geplant, in der niedrigdosierte systemische Steroide mit niedrig dosiertem Theophyllin verglichen werden.

Die therapeutischen Empfehlungen haben sich gegenüber früher nicht wesentlich verändert. Allerdings wurde wegen fehlender Studiendaten die Kombination inhalatives Steroid und lang wirksames Anticholinergikum für die Patientengruppe D gestrichen. Sämtliche Therapieempfehlungen beziehen sich ausschließlich auf die Initialtherapie. Wie die Entwicklung unter einer Langzeittherapie aussieht, soll in großen zukünftigen Studien beobachtet werden. Beispielsweise wird eruiert, wie sich das Weglassen eines Arzneimittels auswirkt.

\section{Aktuelle Publikationen zum neuen GOLD-Algorithmus}

Mithilfe der COPDGene-Kohorte wurde die neue Einteilung überprüft [20]. Wurden die Symptome mithilfe der mMRC-Skala dokumentiert, fielen nur wenige Patienten, nämlich $7,9 \%$, in die Schweregrad-Gruppe C. Bei Verwendung des SGRQ-Lebensqualitätsfragebogens lag der Anteil sogar nur bei 4,9\%. Es scheint also Patienten zu geben, die trotz geringer Symptome ein hohes Mortalitätsrisiko haben.

Zwei sehr gut charakterisierte Populationen mit mehr als 6000 COPD-Patienten stammen aus Kopenhagen [25]. Die mehrdimensionale Stratifizierung ergab plausible Ergebnisse, wenn die Exazerbationsrate der Patienten zugrunde gelegt wurde. Patienten mit kardiovaskulären oder Krebserkrankungen als Komorbiditäten waren häufiger in Gruppe B zu finden als in Gruppe C.
Fazit

Eine Stratifizierung der COPD-Schweregrade allein nach FEV1 ist nicht ausreichend. Dementsprechend wurde in die aktuellen GOLD-Empfehlungen die „Stimme des Patienten“ einbezogen, ebenso wie Komorbiditäten. Das therapeutische Vorgehen ist bisher nicht evidenzbasiert und bezieht sich ausschließlich auf die Initialtherapie. Zukünftig müssen die Klassifizierungsgrenzwerte überprüft werden. Eventuell wird es nötig sein, weitere Subgruppen zu definieren. Auch zukünftig wird die Expertengruppe intensiv an der Optimierung der Empfehlungen arbeiten.

\section{COPD und Endothel}

$\nabla$

\section{Robert Bals, Homburg/Saar}

Die COPD ist eine häufige Erkrankung, die oft mit anderen Krankheiten des Alters vergesellschaftet ist. Bei Älteren kommen hier oft mehrere Komorbiditäten zusammen, was die Differenzialdiagnose und -therapie schwierig macht [26]. Erkrankungen, die oft mit einer COPD zusammen auftreten sind: kardiovaskuläre Krankheiten mit koronarer Herzkrankheit, Schlaganfall, Herzinsuffizienz, Lungenkrebs, Osteoporose, Diabetes oder metabolisches Syndrom [27].

Interessant ist auch ein Blick auf die Todesursachen bei Patienten mit COPD. Patienten mit fortgeschrittener COPD versterben meist im respiratorischen Versagen im Rahmen von Exazerbationen. Patienten mit milderer COPD versterben vor allem an ihren Komorbiditäten, oft am Herzinfarkt oder Lungenkrebs. In epidemiologischen Studien zeigte sich auch, dass eine Einschränkung der Lungenfunktion (FEV1) eng mit einer erhöhten Letalität verbunden ist, oft im Zusammenhang mit kardialen Problemen [28,29]. Diese Komorbiditäten treten überzufällig häufig zusammen auf, sodass gemeinsame Erkrankungsmechanismen vermutet werden. So finden sich bei diesen Erkrankungen oft erhöhte Konzentrationen von Entzündungsmediatoren im Blut. Eine Hypothese besagt, dass die rauch-induzierte Entzündung der Lunge in das Blut und somit den Körper übertritt („spill over“) [30].

\section{Endothelveränderungen bei COPD}

Auch ist das Endothel bei der CODP und vielen ihrer Komorbiditäten in seiner Funktion gestört. Das Endothel kleidet die Gefäße von innen aus und hat eine Vielzahl von physiologischen Funktionen. Eine endotheliale Funktion ist oft bei kardiovaskulären Erkrankungen eingeschränkt und kann mittels der flussabhängigen Gefäßdilatation gemessen werden. Verschiedene Arbeiten zeigen insbesondere eine endotheliale Dysfunktion bei Patienten mit COPD, was auf die enge Verbindung der Lungenerkrankung mit extrapulmonalen Manifestationen hinweist [28].

Auch Reparaturmechanismen des Endothels scheinen bei der COPD gestört zu sein. Endotheliale Progenitorzellen (EPC) sind wohl Knochenmarkszellen, die bei der Reparatur von Gefäßen und eventuell auch anderen Geweben eine Rolle spielen. In zahlreichen Studien fanden sich zumeist verminderte Mengen dieser EPCs im Blut, was allgemein als Einschränkung dieses Reparaturmechanismus verstanden wird [31,32].

Eine neue Hypothese beschreibt einen Mechanismus, wie COPD mit Endothelschäden verbunden sein kann. Es ist gut dokumentiert, dass bei einer COPD die Lunge stark mit Mikroorganismen besiedelt oder infiziert ist [33]. Von mukosalen Oberflächen können Mikroorganismen in die Blutbahn gelangen, was für den Darm gut dokumentiert ist [34]. Gleiches könnte man sich für 
die Lunge auch vorstellen, ohne dass dies durch Studien für die COPD bewiesen wäre. Der neu beschriebene Prozess der „Immunothrombose“ beschreibt die Induktion der Gerinnungskaskade und entzündlicher Endothelveränderungen durch mikrobielle Strukturen, die in der Blutbahn zirkulieren [35]. Über diese Mechanismen könnte ein „spill over“ von Mikroorganimsen in der Blutbahn zu einer Schädigung des Endothels bei der COPD führen.

Zusammenfassend zeigen sich bei der COPD vielfältige Komorbiditäten, die oftmals mit einer Störung der Endothelfunktion assoziiert sind.

\section{COPD und kardiovaskuläre Komorbidität - epidemiologische Evidenz \\ $\nabla$ \\ Winfried J. Randerath, Solingen}

Komorbiditäten können aufgrund gemeinsamer Risikofaktoren entstehen, und sie können zur gegenseitigen Verstärkung von Symptomatik oder Auswirkungen der Grunderkrankungen führen. Mögliche Verbindungen zwischen der COPD und ihren Komorbiditäten sind die systemische Entzündung und die Überblähung als mechanischer Faktor.

\section{Komorbidität und Verlauf}

Komorbiditäten bei COPD finden zunehmend mehr Beachtung. Bei einer telefonischen Befragung berichteten 1003 COPD-Patienten am häufigsten über die Komorbiditäten arterielle Hypertonie (55\%), Hypercholesterinämie (52\%), Depression (37\%), Katarakt (31\%) und Osteoporose (28\%) [36]. In der ECLIPSE-Studie erwiesen sich die kardialen Komorbiditäten als unabhängig vom GOLD-Stadium, und es bestand nur eine schwache Beziehung zum Grad der Flusslimitation.

Komorbiditäten wirken sich bei stationär behandelten Patienten mit Exazerbation maßgeblich auf den weiteren Verlauf aus. So wurden 342 COPD-Patienten mithilfe einer Clusteranalyse in 3 Gruppen eingeteilt: 1) FEV1 38\% vom Soll, 2) FEV1 63\% vom Soll sowie 3) FEV1 58\% vom Soll, verbunden mit zusätzlichen kardiovaskulären Erkrankungen, Adipositas, Diabetes oder Hinweisen auf Inflammation [37]. Die Gruppe 3 hatte sowohl eine höhere Letalität als auch häufigere Krankenhausaufnahmen wegen COPD oder kardiovaskulärer Erkrankungen.

Aus der National Hospital Discharge Survey wurden von 1979 bis 2001 mehr als 47 Millionen stationäre Behandlungen wegen einer COPD ausgewertet, wobei die COPD bei $21 \%$ der Patienten als Hauptdiagnose genannt war [38]. Die Letalität verschiedener Grunderkrankungen war eindeutig höher, wenn zusätzlich eine COPD vorlag.

\section{Koronare Herzerkrankung}

Die Auswertung einer Hausarzt-Datenbank ergab, dass die COPD fünfmal häufiger mit kardiovaskulären Erkrankungen assoziiert war als bei Personen ohne COPD und dass das Risiko eines akuten Myokardinfarkts sogar zehnmal höher war [39]. Nach Exazerbationen betrug die Inzidenz eines Myokardinfarkts in einer anderen Kohorte 1,1 pro 100 Patientenjahre, und statistisch kam ein Myokardinfarkt auf 2513 Exazerbationen [40]. Da Infarkte vor allem während der ersten 5 Tage einer exazerbationbedingten Hospitalisation dokumentiert wurden, kann es sich hier allerdings um eine Überschätzung handeln.
Beeinträchtigungen des Gerinnungssystems wurden in mehreren Studien beschrieben. Bereits im stabilen Zustand bestand eine vermehrte Aggregation der Thrombozyten, die bei COPDExazerbation noch weiter zunahm [41]. Hier könnte eine Verbindung zu häufigeren ischämischen Ereignissen bei Exazerbation bestehen. Raucher mit bronchialer Obstruktion zeigten im Karotis-Ultraschallbefund mehr Hinweise auf eine subklinische Arteriosklerose als Raucher ohne Obstruktion: Plaques wurden häufiger nachgewiesen, und die Intima media hatte einen größeren Durchmesser [42]. Zusätzlich bestand eine Assoziation mit der Höhe des CRPs.

Cerebrovaskuläre Erkrankungen und Herzinsuffizienz Patienten mit COPD hatten ein dreifach erhöhtes Risiko für einen Schlaganfall gegenüber Personen ohne COPD. Dies ergab die Auswertung einer Hausärzte-Datenbank [39]. Die Prävalenz einer Herzinsuffizienz betrug in der ECLIPSE-Studie 7\%, wobei das Ausmaß der Herzinsuffizienz mit stärkerer Flusslimitation zunahm. Eine Analyse aus umgekehrter Sicht, nämlich basierend auf 186 ambulanten Patienten mit Herzinsuffizienz, stellte fest, dass eine zusätzliche COPD bei 39\% der Patienten vorlag [43]. Die Zeit bis zur nächsten Krankenhausaufnahme war bei der COPD-Untergruppe kürzer, ebenso wie die Überlebenszeit. Dies galt vor allem für die COPD-Krankheitsstadien III und IV mit einem 2,1-fach höheren Mortalitätsrisiko. Eine norwegische Forschergruppe wertete ein Register von 4132 Patienten mit Herzinsuffizienz aus [44]. 699 Personen (17\%) waren zusätzlich an COPD erkrankt, und in der Gruppe mit schwerer Herzinsuffizienz waren es sogar 63\%. Die Lungenerkrankung erwies sich als signifikanter unabhängiger Prädiktor der Mortalität. Ob es sich um eine systolische oder eine diastolische Herzinsuffizienz handelte, spielte für das Überleben keine Rolle. Eine Untersuchung aus Großhansdorf zeigte, dass die Größe der Herzhöhlen am stärksten mit dem Quotienten IC/TLC korrelierte, also mit dem Ausmaß der Lungenüberblähung [45]. Die Füllungsbeeinträchtigung des Herzens hängt demnach vor allem vom Ausmaß des Emphysems ab und weniger vom Grad der Obstruktion.

\section{Pulmonale Hypertonie}

Eine geringgradige pulmonale Hypertonie mit mittleren Pulmonalarteriendrucken $>25 \mathrm{mmHg}$ kommt zwar bei schwerer COPD häufiger vor, Drucke über $35 \mathrm{mmHg}$ sind jedoch selten [46]. In den letzten Jahren entstand der Eindruck, dass die Prävalenz der pulmonalen Hypertonie zurückgeht, womöglich im Zusammenhang mit der konsequenteren Langzeitsauerstofftherapie. Berücksichtigt werden muss auch, dass bei mindestens der Hälfte der COPD-Patienten die Echokardiografie keine verwertbaren Aussagen zum Pulmonalarteriendruck erlaubt [47]. Im Vergleich zur Katheter-Untersuchung wird der Druck häufig überschätzt, und nur bei der Hälfte der Patienten waren die Differenzen der mit beiden Methoden gemessenen Drucke geringer als $10 \mathrm{mmHg}$. Computertomografie oder Magnetresonanztomografie sind eindeutig bessere nichtinvasive Methoden als die Echokardiografie [48].

\section{Herzrhythmusstörungen}

Die Prävalenz von Arrhythmien wird bei COPD mit 12-14\% angegeben [49]. Die häufigste Störung ist das Vorhofflimmern. Mit zunehmendem Schweregrad der COPD werden Arrhythmien häufiger beobachtet. Es gibt keine Hinweise auf einen Einfluss der COPD-Medikation. Zur Prävalenz der bei COPD häufig beobachteten Sinustachykardie liegen keine verlässlichen Daten vor. 


\section{Arterielle Hypertonie}

Auswertungen aus den USA zeigen bei schwerer COPD ein erhöhtes Risiko für arterielle Hypertonie und Komorbiditäten wie Diabetes oder kardiovaskuläre Erkrankungen [50]. Je schlechter die Lungenfunktion, desto häufiger waren Hospitalisationen und desto größer war das Mortalitätsrisiko. Welcher pathophysiologische Zusammenhang zwischen COPD und arterieller Hypertonie besteht, wird noch diskutiert. Es könnte sich um eine beschleunigte Alterung handeln, um den Verlust von Bindegewebe oder um eine erhöhte Gefäßsteifigkeit.

\section{Dyslipidämien und Statin-Therapie}

In einer longitudinalen Studie über 10 Jahre wurde der jährliche FEV1-Abfall bei Rauchern, Nichtrauchern und Exrauchern bestimmt [51]. In allen Gruppen war der Verlust an Lungenfunktion geringer, wenn die Personen Statine einnahmen. Kardiovaskuläre Ereignisse wurden unter Statin-Therapie seltener beobachtet. Die prospektive, bevölkerungsbasierte Rotterdam-Kohorte mit knapp 8000 Personen zeigte bei COPD-Patienten mit einer mindestens 2 Jahre durchgeführten Statin-Therapie eine Reduktion der Mortalität um 39\% [52]. Lagen die hs-CRP-Werte über $3 \mathrm{mg} / \mathrm{l}$, wurde die Mortalität durch Statine sogar um $78 \%$ reduziert. Dies weist auf eine Beziehung zur systemischen Inflammation hin. Eine andere Erklärung wäre, dass die medizinische Versorgung der Patienten in der Statin-Gruppe besser war. Die Prävalenz von Dyslipidämien bei COPD unterscheidet sich nicht von der bei anderen Personen. Derzeit wird eine große Studie zu Statinen bei COPD ausgewertet, von der man weitere Erkenntnisse erwarten darf.

\section{Fazit}

Kardiovaskuläre Komorbiditäten sind bei COPD häufig und beeinflussen den Krankheitsverlauf ebenso wie die Überlebensrate. Ein Screening aller COPD-Patienten auf begleitende Herzerkrankungen erscheint sinnvoll, dasselbe gilt umgekehrt für das COPDScreening bei Patienten mit kardiovaskulären Erkrankungen.

\section{COPD-Exazerbation: ein kardiovaskuläres Ereignis? $\nabla$}

\section{Helgo Magnussen, Großhansdorf}

Die Prognose von COPD-Patienten, die wegen einer akuten Exazerbation hospitalisiert werden, wird durch die kardiovaskuläre Komorbidität beeinflusst [53]. Von rund 600 Patienten hatten $32,8 \%$ zusätzlich eine Herzinsuffizienz und 11,6\% einen akuten Myokardinfarkt. Innerhalb von 90 Tagen nach Entlassung verstarb ein signifikant größerer Anteil der Patienten, die diese Begleiterkrankungen aufwiesen.

In einer anderen Studie wurde die Diagnosestellung eines Myokardinfarkts bei 242 Patienten mit COPD-Exazerbation untersucht [54]. Brustschmerzen, EKG-Veränderungen oder erhöhte Troponin-Werte fanden sich bei 124, 122 bzw. 24 Patienten. Von den rund $10 \%$ der Patienten mit erhöhten Troponin-Werten erfüllten fast alle die Definition eines Myokardinfarkts.

\section{Troponin T bei COPD}

Biochemische Marker der kardialen Dysfunktion erlauben eine Einschätzung der Prognose nach akuter COPD-Exazerbation [55]. Im Jahr nach der Exazerbation hatten Patienten mit normalen Werten von Troponin T und NT-proBNP die beste Überlebensrate von rund $90 \%$. Waren beide Parameter erhöht, betrug das kumulative Überleben nur noch $65 \%$. Auch die 30-Tage-Letalität wurde stark durch erhöhte Konzentrationen dieser Biomarker beeinflusst. Die Kombination beider Parameter erlaubte eine bessere Vorhersage als die Verwendung nur eines Markers.

In einer dänischen Untersuchung wurde das kardiale Troponin T bei akuter COPD-Exazerbation gemessen [56]. Erhöhte Werte wurden bei rund $60 \%$ der Patienten nachgewiesen. Über einen Zeitraum von 4 Jahren war das kumulative Überleben am größten bei Patienten mit normalen hs-cTnT-Konzentrationen (unter $14,0 \mathrm{ng} / \mathrm{l}$ ) und am geringsten bei Werten über $40,0 \mathrm{ng} / \mathrm{l}$. Zusätzlich erwies sich eine Tachykardie als ungünstiger prognostischer Faktor.

Dass bei akuter COPD-Exazerbation das kardiale Troponin T gegenüber der stabilen Krankheitsphase ansteigt, wurde in einer aktuellen Studie publiziert [57]. Die Exazerbation war mit einem vierfachen Anstieg des hs-cTnT assoziiert. Bei stabiler COPD wiesen Patienten mit einer FEV1 unter 30\% des Solls die höchsten hs-cTnT-Mittelwerte auf, es bestand also eine Beziehung zum Schweregrad der Erkrankung. Dies war bei akuter Exazerbation nicht der Fall.

Erhöhte Konzentrationen von Troponin sind nicht gleichbedeutend mit einer koronaren Ischämie. Bei Patienten mit normalen Koronararterien können die Serumkonzentrationen erhöht sein durch eine direkte Schädigung der Zellen, zum Beispiel bei Myokarditis, durch eine ungenügende Sauerstoffversorgung oder durch einen erhöhten Sauerstoffbedarf. Bei erhöhter sympathischer Aktivität, bei Tachykardie oder beim Cor pulmonale wird mehr Sauerstoff benötigt.

\section{Herzbelastung durch Lungenüberblähung}

Im Tierexperiment konnte man bei Ratten durch eine Überblähung der Lunge eine kardiale Belastung auslösen [58]. In Großhansdorf untersuchte man bei COPD-Patienten den Einfluss der Überblähung auf die Größe der Herzkammern und der Vorhöfe [45]. Es zeigten sich positive lineare Korrelationen zwischen dem Quotienten IC/TLC und der Fläche des linken bzw. rechten Atriums sowie der Durchmesser der beiden Herzkammern. Außerdem hatten Patienten mit starker Überblähung eine höhere Herzfrequenz in Ruhe.

\section{Bildgebung}

Mit Magnetresonanztomografie untersuchten Autoren aus Rotterdam Lipid-Plaques in den Koronararterien von 253 COPD-Patienten und von 920 Kontrollpersonen [5]. Die Lungenfunktion der Patienten war wenig eingeschränkt (mittlere FEV1 80,5\% des Solls, FEV1/FVC 65,6\%). Koronararterien mit verdickten Wänden wurden bei 54,9\% der Kontrollpersonen nachgewiesen. Bei COPD war dieser Befund noch häufiger, und zwar in Abhängigkeit vom Schweregrad: Die 20 Patienten mit schwerer COPD hatten zu $90 \%$ verdickte Koronararterienwände, bei leichter COPD waren es nur $69 \%$ der Patienten.

Aus den COPDGene- und ECLIPSE-Daten wurde die Häufigkeit von Exazerbationen in Relation zur intrathorakalen Gefäßweite berechnet. Dazu wurde computertomografisch die Weite der Pulmonalarterie und der Aorta bestimmt [59]. War die Pulmonalarterie weiter als die Aorta (Ratio PA: A über 1), war das Risiko zukünftiger schwerer Exazerbationen stark erhöht (Odds ratio $3,44)$.

Bei Exazerbationen müssen auch venöse Thromboembolien differenzialdiagnostisch berücksichtigt werden. Ihre Inzidenz ist bei COPD-Patienten erhöht, und damit ist ein höheres Mortalitätsrisiko verbunden [9]. 


\section{Inflammation und Komorbiditäten}

In einer großen Kohorte aus Kopenhagen mit mehr als 8000 COPD-Patienten wurden Biomarker der Inflammation untersucht [4]. Die Durchschnittswerte des Fibrinogens lagen bei $436 \mathrm{mg} / \mathrm{dl}$, die des hochsensitiven CRPs bei $2,8 \mathrm{mg} / \mathrm{l}$. Patienten mit Komorbiditäten wie Myokardinfarkt, Diabetes oder Pneumonie hatten ein erhöhtes Mortalitätsrisiko, wenn zwei oder drei dieser Entzündungsparameter pathologisch verändert waren.

\section{Inflammation und körperliche Belastung}

In einer aktuellen Untersuchung bestand eine statistisch signifikante Beziehung zwischen Entzündungsparametern und Schweregrad der COPD [4]. Es zeigte sich auch, dass mangelnde körperliche Aktivität mit höheren Konzentrationen der inflammatorischen Marker einherging. Möglicherweise war die wesentliche Ursache für erhöhte Entzündungsmarker im Serum nicht primär die COPD, sondern die körperliche Inaktivität.

Zum Fibrinogen als Biomarker der COPD wurde eine Übersichtsarbeit publiziert, die Studien mit mehr als 40000 Patienten berücksichtigte [60]. Auch hier zeigte sich, dass mehr körperliche Aktivität mit einer besseren FEV1 und geringeren FibrinogenKonzentrationen assoziiert ist.

Pathophysiologische Erkenntnisse legen einen engen Zusammenhang zwischen körperlicher Bewegung und Entzündungsprozessen nahe. Physiologen aus Zürich erforschten die molekularen Vorgänge bei Muskelaktivität und konnten zeigen, dass das Protein PGC- $1 \alpha$ (Peroxisome proliferator-activated receptor $\gamma$ coactivator $1 \alpha$ ) im aktivierten Muskel gebildet wird und eine chronische Entzündungsantwort supprimieren kann [61]. Eine geringgradige Inflammation in den Geweben ist mit der Entwicklung unterschiedlicher chronischer Erkrankungen verbunden, beispielsweise Typ-2-Diabetes, Arteriosklerose, Alzheimer-Demenz oder Morbus Parkinson. Krebserkrankungen wie das Colon- oder das Mamma-Karzinom traten bei körperlich inaktiven Personen häufiger auf.

Die Muskulatur wird seit einigen Jahren als endokrines Organ aufgefasst [62]. Unter Muskelkontraktion steigt das Interleukin6 im Serum bei körperlicher Belastung stark an und beeinflusst beispielsweise den hepatischen Glukosemetabolismus und die Lipolyse im Fettgewebe. Chronische Krankheiten mit reduzierter körperlicher Bewegung gehen daher mit erhöhten Entzündungsparametern einher.

Für die COPD gilt, dass die körperliche Aktivität der stärkste Prädiktor der Gesamt-Mortalität ist [63]. Bestehende Komorbiditäten können sich bereits im stabilen Zustand ungünstig auf die körperliche Aktivität auswirken und auf diesem Weg eine Inflammation begünstigen. Bei Exazerbationen spielt die Zunahme der Lungenüberblähung eine entscheidende Rolle.

\section{Kardiovaskuläre Aspekte der COPD-Pharmakotherapie $\nabla$}

\section{Heinrich Worth, Fürth}

Hinsichtlich der kardialen Auswirkungen der Pharmakotherapie bei COPD ist die Studienlage insgesamt unbefriedigend. Bei prospektiven, randomisierten Studien wurden Patienten mit relevanter kardialer Komorbidität in der Regel ausgeschlossen. Meta-Analysen hatten nicht selten einen Bias bei der Auswahl der Studien, und bei Auswertungen von Patientenregistern bzw. bei Fall-Kontroll-Studien fehlten meist Angaben zum Raucherstatus oder zum Schweregrad der COPD.
Bronchodilatatoren sind diejenige Arzneimittelgruppe, bei der die größten Bedenken hinsichtlich kardiovaskulärer Auswirkungen bestehen. Es fehlen gute randomisierte, kontrollierte Studien bei COPD-Patienten mit symptomatischer Herzerkrankung. Dies erschwert die Beurteilung der kardiovaskulären Sicherheit einer Bronchodilatator-Therapie. Hinzu kommt, dass die Häufigkeit kardiovaskulärer Ereignisse unter Bronchodilatatoren eindeutig altersabhängig ist und zusätzlich von kardiovaskulären Komorbiditäten abhängt.

\section{Beta-2-Sympathomimetika}

Beta-2-Sympathomimetika können auf verschiedenen Wegen unerwünschte kardiale Effekte auslösen. Sie können zu Rhythmusstörungen führen, vor allem zu Sinustachykardien, und sie können die Pumpfunktion des Herzens beeinträchtigen. Eine Hypoxie kann verstärkt und eine Hypokaliämie ausgelöst werden. Insofern besteht bei hohen Dosierungen von Beta-2-Sympathomimetika zweifellos ein Gefährdungspotenzial.

In einer retrospektiven Analyse erhöhte die Vorbehandlung mit hochdosierten Beta-2-Sympathomimetika das Risiko für einen Myokardinfarkt [64]. Nach Einzeldosen von Beta-2-Sympathomimetika wurden Anstiege der Herzfrequenz um etwa 9 Schläge pro Minute beobachtet, und in Langzeitstudien kam es häufiger zu Sinustachykardien, Vorhofflimmern und anderen Rhythmusstörungen [65].

Unter Formoterol wurden bei COPD-Patienten ohne kardiale Komorbiditäten in einer achtwöchigen Studie häufiger Arrhythmien und ventrikuläre Tachykardien beobachtet als unter Plazebo [66]. In einer Analyse von 17 Studien mit Salmeterol war die Inzidenz kardiovaskulärer Ereignisse bei kardial vorerkrankten COPD-Patienten zwar insgesamt höher als bei nicht herzkranken Patienten, jedoch fand man keinen Unterschied zwischen den mit Salmeterol oder mit Plazebo behandelten Studienteilnehmern. Patienten mit vorbestehender Herzinsuffizienz und einer Ejektionsfraktion unter $45 \%$ wurde in einer anderen Studie untersucht [67]. Der vermehrte Gebrauch kurzwirksamer Beta-2Sympathomimetika war mit einem etwas höheren Risiko für Hospitalisationen wegen Herzinsuffizienz assoziiert. Die Therapie mit Salmeterol, und zwar sowohl als Monotherapie als auch kombiniert mit Fluticason, wurde in der TORCH-Studie mit Plazebo verglichen [68]. Die Zahl der Todesfälle war in allen Behandlungsgruppen ähnlich, sodass die Salmeterol-Inhalation nicht mit einer erhöhten Sterblichkeit assoziiert war.

\section{Anticholinergika}

Für die Anticholinergika-Therapie gibt es sowohl Hinweise für eine positive als auch für eine negative Assoziation mit kardiovaskulären Ereignissen [69]. In der Lung Health Study wurden zwar für Ipratropiumbromid häufigere kardiovaskuläre Ereignisse sowie eine erhöhte Rate an supraventrikulären Tachykardien beobachtet [70]. Allerdings gab es Probleme mit unzureichender Compliance der Patienten, und letztendlich konnte kein definitiver Zusammenhang mit Ipratropiumbromid hergestellt werden. Eine Metaanalyse von 17 randomisierten kontrollierten Studien mit Tiotropium oder Ipratropiumbromid sorgte für Aufsehen, denn bei Patienten mit Inhalation von Anticholinergika wurde ein relatives Risiko von 1,58 für Tod, Herzinfarkt oder Schlaganfall berechnet [71]. Diese Endpunkte der Metaanalyse waren jedoch in den zugrunde gelegten Publikationen keine Studienendpunkte gewesen. Außerdem fehlten Angaben zum Schweregrad der COPD. Auch andere Kritikpunkte an dieser Metaanalyse wurden geäußert. 
Kardiovaskuläre Effekte von Ipratropiumbromid wurden bei mehr als 80000 COPD-Patienten mit neu diagnostizierter COPD analysiert [72]. Dokumentiert wurden mehr als 6000 Ereignisse wie Arrhythmien, akutes Koronarsyndrom oder Herzinsuffizienz. Die Inhalation mit dem Anticholinergikum war mit einem 29\% höheren Risiko für stationäre Aufnahmen wegen kardiovaskulärer Ereignisse assoziiert. Unter der langfristigen Behandlung mit Tiotropium in der UPLIFT-Studie zeigte sich hingegen eine geringere kumulative Häufigkeit von kardiovaskulären Ereignissen bzw. Todesfällen [73]. Allerdings waren Patienten mit erhöhtem kardiovaskulärem Risiko von der Studienteilnahme ausgeschlossen, und die klinische Prüfung diente primär dem Nachweis der Wirksamkeit und nicht der Sicherheit des Arzneimittels. Eine Gegenüberstellung von 29 gepoolten Tiotropium-Studien mit der UPLIFT-Studie zur kardiovaskulären Sicherheit ergab, dass das relative Risiko für Schlaganfall, Herzinfarkt oder kardiovaskulären Tod in der UPLIFT-Studie geringer war [74].

Eine vergleichende Analyse zu den Medikamenten Indacaterol, Formoterol, Salmeterol und Tiotropium zeigte gegenüber Plazebo zwar leichte Effekte hinsichtlich der Anzahl von Patienten, deren QTc-Dauer gegenüber dem Ausgangswert angestiegen war, diese Veränderungen wurden jedoch nicht als klinisch relevant eingeschätzt [75].

In zwei zusammenfassend ausgewerteten Studien ergab sich ein leicht erhöhtes Risiko für Angina pectoris und Tod, wenn Tiotropium aus dem Respimat ${ }^{\circledR}$ inhaliert worden war. Jedoch war der Effekt nicht statistisch signifikant [76].

Aus Kanada liegen zwei Fall-Kontroll-Studien zu Arrhythmien vor, die zur Krankenhausaufnahme führten $[77,78]$. In der Saskatchewan-Kohorte kam es insgesamt zu 1,4 Arrhythmien pro 100 Patientenjahren, und unter Ipratropiumbromid ebenso wie unter langwirksamen Beta-2-Sympathomimetika war das relative Risiko für Rhythmusstörungen erhöht. Die zweite Analyse aus Quebec hatte ähnliche Ergebnisse. Ein kritischer Kommentar zu diesen Publikationen stellte fest, dass zur Erforschung der kardiovaskulären Sicherheit der Bronchodilatator-Therapie prospektive Studien bei COPD-Patienten mit und ohne kardiale Vorerkrankungen unbedingt erforderlich sind [79].

\section{Theophyllin, Glukokortikoide und Roflumilast}

Lebensbedrohliche Theophyllin-Intoxikationen wurden in einer prospektiven Analyse longitudinal dokumentiert [80]. Über einen 10-Jahres-Zeitraum hatten bei Serumkonzentrationen über $30 \mathrm{mg} / 1$ 45\% der Patienten akute und $40 \%$ chronische Intoxikationen.

Bei systemischer Gabe von Glukokortikoiden sind unerwünschte Wirkungen wie Blutdruckanstieg, Flüssigkeitsretention, Hypokaliämie und Anstieg des Blutzuckers möglich. Dies sind Gründe dafür, orale systemische Glukokortikoide bei herzkranken COPDPatienten zurückhaltend einzusetzen. Das Risiko eines Vorhofflimmerns war oberhalb einer Tagesdosis von 7,5 mg Prednison auf fast das Fünffache erhöht [81].

Unter Roflumilast traten im Vergleich zu Plazebo keine gehäuften Herzrhythmusstörungen auf. In einer gepoolten Analyse mit etwa 12000 COPD-Patienten war die Wahrscheinlichkeit für den kombinierten Endpunkt Myokardinfarkt, Schlaganfall oder kardiovaskuläre Sterblichkeit über eine Behandlungszeit von 12 Monaten unter Roflumilast signifikant geringer als unter Plazebo [82].

\section{Fazit}

Bei Patienten mit COPD ohne zusätzliche Herzerkrankung ist die kardiovaskuläre Sicherheit von Anticholinergika, Beta-2-Sympathomimetika und Roflumilast gegeben. Inhalative Kortikosteroide und Roflumilast bringen kein kardiovaskuläres Risiko mit sich. Bei Patienten mit kardialer Komorbidität können unter LABAs oder lang wirksamen Anticholinergika unerwünschte kardiale Effekte auftreten. Von allen Bronchodilatatoren zeigt Theophyllin das ungünstigste Nutzen-Risiko-Verhältnis.

\section{COPD - Die Beta-Blocker-Story}

$\nabla$

\section{Michael Pfeifer, Regensburg}

Bei niedergelassenen Ärzten bestehen teilweise noch Vorbehalte gegenüber der Verordnung von Betablockern bei COPD-Patienten. Dies zeigte eine aktuelle Auswertung der SHIFT-Studie [83], in der Patienten mit COPD-Anamnese deutlich seltener eine Therapie mit Betablockern erhielten. Dass Sorgen vor unerwünschten Wirkungen unbegründet sind, wurde in mehreren Cochrane-Reviews dargestellt [65]. Kardioselektive Betablocker zeigten in Meta-Analysen keine ungünstigen Auswirkungen auf den Verlauf der FEV1 bei chronisch obstruktiver Lungenerkrankung.

\section{Betablocker und Mortalität}

Die erste Auswertung, die an einer großen Kohorte den günstigen Effekt von Betablockern nach Myokardinfarkt zeigte, wurde 1998 publiziert [84]. Ausgehend von mehr als 200000 Patienten, die nach akutem Infarkt nachhause entlassen wurden, analysierte man unter anderem auch 41814 Patienten mit zusätzlicher COPD. Unter Betablocker-Therapie war die Überlebensrate in den folgenden 24 Monaten nicht nur im Gesamtkollektiv günstiger, sondern auch bei COPD. Bei der Interpretation der Studienergebnisse muss man jedoch berücksichtigen, dass es sich lediglich um eine deskriptive Auswertung handelte und dass keine Adjustierung erfolgte

Eine europäische Studie mit Valsartan bei mehr als 14000 Patienten mit akutem Myokardinfarkt zeigte eindrücklich die schlechtere Prognose von Patienten mit COPD im Vergleich zu den übrigen Patienten [85]. Die Therapie mit Betablockern verringerte die kumulative Todesrate in allen Gruppen signifikant und klinisch relevant, sodass ein positiver Therapieeffekt darstellbar war.

Ein anderes Patientenkollektiv wurde nach einer Gefäßoperation longitudinal weiter beobachtet [86]. Auch diese Patienten mit Arteriosklerose profitierten von einer Therapie mit Betablockern. Sowohl die 30-Tage- als auch die Langzeitmortalität waren signifikant geringer. Dies galt gleichermaßen für die 39\% Patienten mit COPD, von denen gut ein Drittel unter einer Betablockerbehandlung stand.

Die Wirkung von Betablockern im Vergleich zu anderen antihypertensiven Medikamenten war Thema einer amerikanischen Studie [87]. Beim Patientenkollektiv fiel die geringe Zahl von Frauen auf (nur 2,5\%), die auf die Auswahl der Studienzentren, nämlich Veterans Affairs Medical Centers, zurückgeht. Ein weiteres Einschlusskriterium war eine mindestens $90 \%$ ige Adhärenz gegenüber der antihypertensiven Therapie. Im Vergleich zu Kalziumkanalblockern senkten Betablocker die Mortalität bei COPD erheblich. Die Auswertung einer Subgruppe von Patienten, die in den vorausgegangenen 6 Monaten Bronchodilatatoren inhaliert hatten, kam zu demselben Ergebnis. 


\section{Betablocker und Exazerbationen}

Wenn COPD-Patienten bei akuter Exazerbation hospitalisiert wurden, hatten Personen mit Betablocker-Therapie eine geringere Mortalität. Dies ergab eine Auswertung von Dransfield 2008 an 825 Patienten [88]. Eine Analyse von mehr als 35000 Patienten mit akuter COPD-Exazerbation und gleichzeitiger Herzerkrankung zeigte hingegen keine Schutzwirkung der Betablockertherapie hinsichtlich der stationären Wiederaufnahme nach Entlassung, einer erforderlichen Beatmung oder der Mortalität [89]. Untersucher aus Schottland werteten eine Datenbank von knapp 6000 Patienten über eine Dauer von 10 Jahren aus [90]. Dabei ging es um Medikamentenkombinationen, die typischerweise bei COPD-Patienten verordnet werden, und den Einfluss einer zusätzlichen Betablockertherapie. Unter einer Behandlung mit Betablockern war die Überlebensrate insgesamt um $22 \%$ besser, und der günstige Effekt war bei diversen Medikamenten-Kombinationen sichtbar. Auch Exazerbationen, Myokardinfarkte und Krankenhausaufnahmen wegen respiratorischer Ereignisse kamen innerhalb von 10 Jahren seltener vor.

\section{Mögliche Wirkmechanismen}

Dass Betablocker bei COPD das kardiale Risiko senken, könnte an einer Reduktion der Herzfrequenz und der günstigen Wirkung auf den erhöhten Sympathotonus liegen. Ebenfalls diskutiert werden krankheitsspezifische Effekte, zum Beispiel eine antioxidative Wirkung oder eine Verbesserung der Effizienz der Betamimetika durch Sensibilisierung des Betarezeptors.

Naheliegend ist der günstige Einfluss auf die Herzfrequenz. Patienten mit schwerer COPD haben zwar meist keine explizite Tachykardie, jedoch eine höhere Herzfrequenz als leicht erkrankte Personen [91]. Eine statistische Beziehung zwischen Herzfrequenz in Ruhe und Lebenserwartung wurde dargestellt: Im GOLD-Stadium III/IV war die mediane Lebenserwartung um 5 Jahre kürzer, wenn die Herzfrequenz höher als 85 pro Minute lag, als wenn sie weniger als 65 Schläge pro Minute betrug. Die Substanz Ivabradin wurde in der SHIFT-Studie bei Patienten mit Herzinsuffizienz zusätzlich zu Betablockern verordnet [83]. Das Verum senkte die Herzfrequenz um etwa 10 Schläge pro Minute gegenüber Plazebo signifikant. Damit verbunden war eine Verbesserung der Überlebenszeit.

\section{Fazit}

Betablocker scheinen auch die Mortalität von COPD-Patienten im Langzeitverlauf zu verringern, und zwar nach Myokardinfarkt, nach gefäßchirurgischen Eingriffen und bei Exazerbationen. Krankenhausaufnahmen und Exazerbationen kommen unter dieser Therapie statistisch seltener vor. Allerdings muss man bei der Interpretation der vorhandenen Daten beachten, dass es sich ausnahmslos um retrospektive Auswertungen handelt. Teilweise erfolgte eine sehr spezielle Selektion der Patienten, und nicht immer waren zuverlässige Informationen über Vorbehandlungen vorhanden. Daten zu einer Dosis-Wirkungs-Beziehung gibt es nicht.

In prospektiven Studien sollte bei COPD-Patienten überprüft werden, welchen Einfluss die medikamentöse Senkung der Herzfrequenz auf Mortalität und Exazerbationen hat.

\section{Das COSYCONET: Design und Herausforderungen \\ $\nabla$}

\section{Tobias Welte, Hannover}

\section{Das COSYCO-Netzwerk}

Die Abkürzung COSYCONET steht für German COPD and Systemic Consequences - Comorbidities Network. In diesem Netzwerk wollen die Forscher bei COPD-Patienten die Häufigkeit extrapulmonaler Erkrankungen erfassen, Beziehungen zur systemischen Inflammation analysieren und Konsequenzen hinsichtlich Morbidität, Letalität und Krankheitskosten dokumentieren [92]. Das Konsortium besteht aus 7 Universitätskliniken, 22 Studienzentren sowie dem Helmholtz-Zentrum München und dem MaxPlanck-Institut in Bad Nauheim. Sprecher des Netzwerkes ist Professor Claus Vogelmeier aus Marburg, der das Projekt maßgeblich auf den Weg gebracht hat.

Fördermittel für das Projekt wurden zunächst für 3 Jahre bewilligt. 2012 wurde der Verlängerungsantrag für weitere 3 Jahre vom BMBF genehmigt. Nach Ablauf der Förderung durch das BMBF ist beabsichtigt, die Kohorte im Rahmen des Deutschen Zentrums für Lungenforschung weiterzuführen [93].

\section{Aufbau der COPD-Kohorte und Herausforderungen}

Im Teilprojekt 1 wird seit 2010 eine nationale Kohorte von COPDPatienten aufgebaut und langfristig weiter verfolgt. Diese Patientengruppe soll als Basis für unterschiedliche Spezialstudien dienen. Rekrutiert werden 3000 Patienten mit allen Schweregraden der COPD. Es wird ein Patientenregister erstellt, und die Daten der COSYCONET-Studienteilnehmer werden mit denen von populationsbasierten Kontrollkohorten wie der Augsburger KORAoder der Greifswalder SHIP-Kohorte verglichen. Ein wesentliches Element des Projektes ist der Aufbau einer Biobank mit Blut- und Gewebeproben der COPD-Patienten. Damit können Konsequenzen der systemischen Inflammation genauer evaluiert werden. Unterschiedliche Teilprojekte und Tiermodelle erforschen beispielsweise die Endothelfunktion, die pulmonale Gefäßbiologie, systemische und pulmonale Reparatur- und Regenerationsmechanismen oder die systemischen Effekte pulmonaler Infektionen. Auch gesundheitsökonomische Analysen sind geplant. Medikamentöse oder andere therapeutische Interventionen sind nicht vorgesehen, und in die bestehende Therapie des Patienten wird nicht eingegriffen.

Geplant war, dass in 20 Zentren pro Jahr jeweils etwa 50 bis 60 COPD-Patienten in die Kohorte aufgenommen werden können. Von den ursprünglich rekrutierten Studienzentren waren jedoch nicht alle in der Lage, die vereinbarten Patientenzahlen bereitzustellen. Der erforderliche personelle Aufwand in den Studienzentren ist immens. Pro Visite werden 3 bis 4 Stunden einkalkuliert. Insgesamt ist die Finanzierung der Studie knapp kalkuliert, für kleinere Studienzentren, die bisher nicht über eine Studienambulanz verfügen und die erst Infrastrukturen aufbauen müssen, reicht die Finanzierung nicht aus. Zwar konnten im Rahmen sogenannter „unrestricted grants“ zusätzliche Mittel aus der pharmazeutischen Industrie eingeworben werden, die Rekrutierung verzögerte sich jedoch aus genannten Gründen.

\section{Studienendpunkte}

Die Studienendpunkte beinhalten Lungenfunktionsuntersuchungen wie Spirometrie, Bodyplethysmografie und die Messung der Dlco. Außerdem führen die Patienten einen 6-Minuten-Gehtest durch. Die Körperzusammensetzung wird mit bioelektrischer Impedanzanalyse (BIA) gemessen. Es werden Blutproben ent- 
nommen und Blutgasanalysen durchgeführt. Kardiovaskuläre Untersuchungen beinhalten Ruhe-EKG und Echokardiografie sowie optional die Bestimmung der Karotiden-Intima-MediaDicke. In der zweiten Förderperiode werden zusätzlich bei ausgewählten Patienten Untersuchungen zur Schlafqualität und zur Besiedlung der Atemwege mit Bakterien durchgeführt. Als bildgebende Methoden werden thorakale HR-CTs durchgeführt. Zusätzlich müssen die Patienten Fragebögen zu unterschiedlichen Facetten der Erkrankung beantworten.

\section{Standardisierung und Datenqualität}

Die Studiengruppe hat große Anstrengungen unternommen, die diagnostischen Verfahren gut zu standardisieren. Dabei mussten diverse Probleme bewältigt werden. So zeigte sich, dass alte und neue Geräte zur Bodyplethysmografie bei ein und demselben Patienten zu unterschiedlichen Ergebnissen führten, mit klinisch relevanten Differenzen von bis zu $400 \mathrm{ml}$ beim TGV.

Die Befundung der HR-CTs erfolgt als zentralisierte Auswertung durch Experten um Prof. Kauzcor aus Heidelberg. Bei der Bildgebung ist zu erwarten, dass neuere Geräte in der Zukunft mehr pathologische Veränderungen darstellen als heutige HR-CTs. Dies wird bei der Interpretation der Studienergebnisse zu berücksichtigen sein.

Beim Aufbau der Biobank stieß man auf ungeklärte juristische Fragen hinsichtlich der Rechte der Patienten, der Ärzte als „Sammler“ der Materialien und der Wissenschaftler in den Forschungsinstituten. Inzwischen konnte in Zusammenarbeit mit der Technologie- und Methodenplattform für die vernetzte medizinische Forschung (TMF) e.V. jedoch eine tragfähige Lösung gefunden werden.

Die Datenqualität dieser Kohorte wird herausragend sein. Teilbereiche wie die ausführliche kardiologische Diagnostik oder die standardisierte Untersuchung des Schlafes wurden bisher nicht in dieser Form in anderen Kohorten untersucht. Zweifellos werden aus dem Projekt wichtige Erkenntnisse hervorgehen.

\section{Tiotropium beim Asthma bronchiale und das TinA-Studienprogramm \\ $\nabla$}

\section{Christian Taube, Leiden, NI}

In den aktuellen Leitlinien zur Behandlung von Asthma werden langwirksame Anticholinergika bisher nicht erwähnt. Dieses beruht im Wesentlichen auf der Wahrnehmung, dass kurzwirksame Anticholinergika im direkten Vergleich mit kurzwirksamen Beta-Sympathomimetika in kurzen Studien meist eine geringere Wirksamkeit auf die Änderung der Lungenfunktion aufwiesen [94]. Diese Ergebnisse führten dazu, dass Anticholinergika lange Zeit nicht in der Dauertherapie bei Patienten mit Asthma berücksichtigt wurden. Basierend auf aktuellen Studiendaten und Entwicklungen, kann aber in Zukunft diesbezüglich eine Änderung erwartet werden.

\section{Vergleich zwischen langwirksamen Beta-Sympatho- mimetika und Anticholinergika}

In 2010 wurde eine Studie des amerikanischen Asthmanetzwerkes über die Wirksamkeit des langwirksamen Anticholinergikums Tiotropium im direkten Vergleich mit einer Verdoppelung der Steroiddosis oder einem langwirksamen Beta-Sympathomimetikum (Salmeterol) veröffentlicht. Bei Patienten mit unkontrolliertem Asthma, die zweimal täglich mit $80 \mu \mathrm{g}$ BDP inhaliert hatten, wurde der Effekt von einmal $18 \mu \mathrm{g}$ Tiotropium verglichen mit dem von zweimal $50 \mu \mathrm{g}$ Salmeterol bzw. einer Verdoppelung der Steroiddosis auf $2 \times 160 \mu$ g BDP [95]. Nach einer Therapiedauer von 14 Wochen blieb der Peak flow unter der erhöhten Steroidedosis unverändert. Dagegen verbesserte sowohl das langwirksame Beta-Sympathomimetikum (LABA) als auch das langwirksame Anticholinergikum (LAMA) die Peak-flow-Werte am Morgen und am Abend signifikant, wobei der Anstieg unter Tiotropium sogar numerisch etwas größer war. Diese Studie sorgte für erhebliches Aufsehen, u.a. deswegen, weil sie nicht durch ein pharmazeutisches Unternehmen gesponsert worden war.

Um die Wirksamkeit von Tiotropium weiter zu untersuchen, wurden Patienten mit mittelgradigem persistierenden Asthma in einer Proof-of-concept-Studie mit Tiotropium oder mit Salmeterol behandelt [96]. Während der vierwöchigen Run-in-Phase erhielten alle Studienteilnehmer 400 bis $1000 \mu \mathrm{g}$ Budesonid und zusätzlich zweimal täglich $25 \mu \mathrm{g}$ Salmeterol. Anschließend erfolgte die Randomisierung auf entweder $5 \mu \mathrm{g}$ Tiotropium am Abend oder die Weiterführung der Salmeterol-Therapie $(2 \times 25 \mu \mathrm{g})$ oder Plazebo. Die Behandlungsdauer betrug 16 Wochen. Unter Plazebo, also ohne das in der Run-in-Phase inhalierte Salmeterol, fiel der mittlere Morgen-Peak-flow um 20-25 ml gegenüber dem Ausgangswert ab. Unter Tiotropium blieben die Werte dagegen stabil, ebenso wie unter Salmeterol. Beide Medikamente zeigten gemessen am Peak flow eine ähnlich gute Wirksamkeit.

\section{TinA-Studienprogramm}

Ein weiterer wichtiger Ansatzpunkt für den Einsatz von Anticholinergika liegt bei Patienten mit schwerem Asthma, für die nur wenige Behandlungsoptionen existieren. Von Patienten mit COPD ist bekannt, dass die gleichzeitige Gabe von Anticholinergika und Beta-Sympathomimetika synergistisch auf die Lungenfunktion wirkt. Die Arbeitsgruppe um Kerstjens aus Groningen führte daher bei schwerem Asthma eine Studie mit Tiotropium als Add-on-Therapie durch [97]. Zusätzlich zur Therapie mit inhalativen Steroiden und langwirksamen Beta-Sympathomimetika erhielten die Patienten morgens Tiotropium in einer Tagesdosis von entweder $5 \mu$ g oder $10 \mu$ g. Der dritte Studienarm bestand aus der Gabe von Plazebo. In einem komplizierten Crossover-Design inhalierten die Patienten jede der 3 Behandlungen über jeweils 8 Wochen. Die 107 Studienteilnehmer wiesen zu Beginn eine starke bronchiale Obstruktion auf mit prä- und postbronchodilatatorischen FEV1-Werten von nur 58\% bzw. 65\% des Solls. Nach 8 Wochen zeigte sich bei der Messung der FEV1 über 24 Stunden eine Zunahme der Lungenfunktion mit einer Dosierung von $5 \mu \mathrm{g}$ pro Tag. Eine Erhöhung der Dosis auf $10 \mu \mathrm{g}$ hatte keinen zusätzlichen Effekt. Beide Dosierungen von Tiotropium waren dem Plazebo klar überlegen.

Im nächsten Schritt wurden mehr als 900 Patienten mit schwerem, schlecht kontrolliertem Asthma bronchiale im Rahmen von zwei identischen Studien untersucht [98]. Die Studienteilnehmer hatten trotz einer hochdosierten Therapie mit inhalativen Steroiden $(\geq 800 \mu \mathrm{g}$ Budesonid-Äquivalent) und der Inhalation eines LABAs eine persistierende Obstruktion mit einer post-bronchodilatatorischen FEV1 unter $80 \%$ des Solls. Beim Screeningbesuch waren Asthmasymptome vorhanden (ACQ $\geq 1,5)$, und im vorausgegangenen Jahr hatten die Patienten mindestens eine AsthmaExazerbation durchgemacht. Rund 60\% der Studienteilnehmer waren Frauen, das mittlere Alter lag bei 53 Jahren, und das Asthma hatte durchschnittlich mit 26 Jahren begonnen. Die primären Endpunkte nach 24-wöchiger Therapie waren die Tal- und die 
Spitzen-FEV1-Werte, gemessen jeweils direkt vor bzw. 3 Stunden nach Inhalation von $5 \mu$ g Tiotropium bzw. Plazebo. Gegenüber den Ausgangswerten führte Tiotropium zu einem signifikanten Anstieg der beiden FEV1-Werte um durchschnittlich ca. 150 bzw. $350 \mathrm{ml}$, während unter Plazebo keine signifikanten Verbesserungen beobachtet wurden. Ein weiterer primärer Endpunkt war die Zeit bis zum Auftreten der ersten Asthma-Exazerbation. Hier ergab sich durch Tiotropium eine $21 \%$ ige Reduktion des Risikos gegenüber Plazebo $(p=0,03)$. Die Anzahl der notwendigen Behandlungen (number needed to treat) zur Vermeidung einer Exazerbation betrug 15. Diese Daten zeigen eindrücklich, dass bei Patienten mit schwerem Asthma und maximaler inhalativer Standardtherapie durch eine Add-on-Behandlung mit Tiotropium sowohl eine Besserung der Lungenfunktion als auch die Reduktion der Exazerbationen erreicht werden kann. Warum Anticholinergika einen solchen Effekt auf Exazerbationen haben, ist aber zum jetzigen Zeitpunkt nicht bekannt.

\section{Mögliche Mechanismen für die Wirksamkeit von Anticholinergika beim Asthma}

Rezeptoren für Acetylcholin befinden sich nicht nur auf der glatten Muskulatur, sondern auch auf Atemwegsepithelzellen, Fibroblasten und auf Entzündungszellen [99]. Acetylcholin beeinflusst beispielsweise die Mukussekretion in den Atemwegen, ein auch für Asthma relevanter Effekt. Das von Epithelzellen produzierte Acetylcholin moduliert sensorische Nervenfasern und beeinflusst pro- und antiinflammatorische Zytokine.

In Mausmodellen ließ sich nachweisen, dass Tiotropium die Spätreaktion nach Allergenprovokation blockiert [100]. Eine Arbeitsgruppe aus Groningen erforschte die allergeninduzierte Inflammation und wies nach, dass Tiotropium dabei ähnlich gut wirksam ist wie Budesonid [101]. Diese Befunde sprechen dafür, dass für die Wirksamkeit von Tiotropium bei Asthma andere Mechanismen als nur die Bronchodilatation relevant sind. Weitere Untersuchungen sind notwendig, um diese zugrundeliegenden Mechanismen aufzuklären.

\section{Unerwünschte Wirkungen}

In den klinischen Studien zu Tiotropium wurde der Respimat ${ }^{\circledR}$ zur Inhalation verwendet. Zwei aktuelle Publikationen thematisierten ein erhöhtes Letalitätsrisiko bei Verwendung dieses Devices $[98,102]$. In dieser Patientenpopulation mit Asthma ist zu konstatieren, dass Tiotropium eine sichere Medikation ohne wesentliche unerwünschte Ereignisse ist. Insbesondere im Punkt kardiale Ereignisse wurde unter Tiotropium kein erhöhtes Risiko im Vergleich zu Plazebo-behandelten Patienten gefunden. Es muss aber berücksichtigt werden, dass in den TinA-Studien schwerwiegende Begleiterkrankungen ein Ausschlusskriterium waren, sodass die Ergebnisse nicht automatisch auf Patienten mit kardialen Vorerkrankungen extrapoliert werden können.

\section{Interessenkonflikt}

\section{$\nabla$}

J. Lorenz erhielt Erstattungen von Reisekosten oder Teilnahmegebühren für Kongresse oder Fortbildungsveranstaltungen sowie Honorare für Vorträge.

H. Magnussen erhielt Erstattungen von Reisekosten oder Teilnahmegebühren für Kongresse oder Fortbildungsveranstaltungen, Honorare für Vorträge, Unterstützung für Forschungsvorhaben oder finanzielle Zuwendungen für die Teilnahme an einer Studie, Finanzierung von Mitarbeitern sowie Honorare für Beratertätigkeit.
M. Pfeifer erhielt Erstattungen von Reisekosten oder Teilnahmegebühren für Kongresse oder Fortbildungsveranstaltungen, Honorare für Vorträge, Unterstützung für Forschungsvorhaben oder finanzielle Zuwendungen für die Teilnahme an einer Studie sowie Honorare für Beratertätigkeit.

W. Randerath erhielt Erstattungen von Reisekosten oder Teilnahmegebühren für Kongresse oder Fortbildungsveranstaltungen, Honorare für Vorträge, Unterstützung für Forschungsvorhaben oder finanzielle Zuwendungen für die Teilnahme an einer Studie sowie Honorare für Beratertätigkeit.

G. Steinkamp erhielt Erstattungen von Reisekosten oder Teilnahmegebühren für Kongresse oder Fortbildungsveranstaltungen sowie Honorare für Medical Writing.

H. Teschler erhielt Erstattungen von Reisekosten oder Teilnahmegebühren für Kongresse oder Fortbildungsveranstaltungen sowie Honorar für einen Vortrag.

C. Vogelmeier erhielt Erstattungen von Reisekosten oder Teilnahmegebühren für Kongresse oder Fortbildungsveranstaltungen, Honorare für Vorträge, Unterstützung für Forschungsvorhaben oder finanzielle Zuwendungen für die Teilnahme an einer Studie sowie Honorare für Beratertätigkeit.

T. Welte erhielt Honorare für Vorträge, Unterstützung für Forschungsvorhaben oder finanzielle Zuwendungen für die Teilnahme an einer Studie sowie Honorare für Beratertätigkeit.

R. Bals, C. Taube und H. Worth geben an, dass kein Interessenkonflikt besteht.

\footnotetext{
Institute

${ }^{1}$ Klinik für Pneumologie und Internistische Intensivmedizin, Klinikum Lüdenscheid

2 Pneumologie, Allergologie, Beatmungsmedizin, Universitätsklinikum des Saarlandes

${ }^{3}$ Pneumologisches Forschungsinstitut an der LungenClinic Grosshansdorf

${ }^{4}$ Klinik Donaustauf, Universität Regensburg

${ }^{5}$ Klinik für Pneumologie und Allergologie, Krankenhaus Bethanien, Solingen

${ }^{6}$ Medizinisch-wissenschaftliches Publizieren, Schwerin

${ }^{7}$ Department of Pulmonology, Leiden University Medical Center,

The Netherlands

${ }^{8}$ Ruhrlandklinik, Westdeutsches Lungenzentrum, Universitätsklinikum Essen

${ }_{9}^{9}$ Pneumologie im Universitätsklinikum Giessen und Marburg, Standort Marburg

${ }^{10}$ Klinik für Pneumologie, Medizinische Hochschule Hannover

${ }^{11}$ Medizinische Klinik I, Klinikum Fürth
}

\section{Literatur}

1 Kosacz NM. Chronic obstructive pulmonary disease among adults United States, 2011. MMWR Morb Mortal Wkly Rep 2012; 61: 938 943

2 Schnell $K$, Weiss CO, Lee $T$ et al. The prevalence of clinically-relevant comorbid conditions in patients with physician-diagnosed COPD: a cross-sectional study using data from NHANES 1999-2008. BMC Pulm Med 2012; 12: 26

3 Agusti A, Edwards LD, Rennard SI et al. Persistent systemic inflammation is associated with poor clinical outcomes in COPD: a novel phenotype. PLoS One 2012; 7: e37483

4 Thomsen M, Dahl M, Lange P et al. Inflammatory biomarkers and comorbidities in chronic obstructive pulmonary disease. Am J Respir Crit Care Med 2012; 186: $982-988$

5 Lahousse L, van den Bouwhuijsen OJ, Loth DW et al. Chronic obstructive pulmonary disease and lipid core carotid artery plaques in the elderly: the Rotterdam Study. Am J Respir Crit Care Med 2013; 187: $58-64$

6 Sin $D D$, MacNee $W$. Chronic obstructive pulmonary disease and cardiovascular diseases: a "vulnerable" relationship. Am J Respir Crit Care Med 2013; 187: 2-4

7 Suissa S, Dell'Aniello S, Ernst P. Long-term natural history of chronic obstructive pulmonary disease: severe exacerbations and mortality. Thorax 2012; 67: 957-963 
8 Steer J, Norman EM, Afolabi OA et al. Dyspnoea severity and pneumonia as predictors of in-hospital mortality and early readmission in acute exacerbations of COPD. Thorax 2012; 67: 117-121

9 Bertoletti L, Quenet S, Mismetti P et al. Clinical presentation and outcome of venous thromboembolism in COPD. Eur Respir J 2012; 39: $862-868$

10 Wouters EF, Bredenbroker D, Teichmann P et al. Effect of the phosphodiesterase 4 inhibitor roflumilast on glucose metabolism in patients with treatment-naive, newly diagnosed type 2 diabetes mellitus. J Clin Endocrinol Metab 2012; 97: E1720-E1725

11 Buhl R, Welte T, Vogelmeier $C$ et al. Evidenz fur eine frühzeitige Therapie der COPD mit Tiotropium [Early treatment of COPD with tiotropium]. Pneumologie 2012; 66: 589-595

$12 \mathrm{Kim}$ V, Kretschman DM, Sternberg AL et al. Weight gain after lung reduction surgery is related to improved lung function and ventilatory efficiency. Am J Respir Crit Care Med 2012; 186: 1109-1116

13 Global Initiative for Chronic Obstructive Lung Disease. www.goldcopd.org. 2013

14 Mannino DM, Sonia BA, Vollmer WM. Chronic obstructive pulmonary disease in the older adult: what defines abnormal lung function? Thorax 2007; 62: 237-241

15 Mannino DM, az-Guzman E. Interpreting lung function data using $80 \%$ predicted and fixed thresholds identifies patients at increased risk of mortality. Chest 2012; 141: 73-80

16 Tashkin DP, Celli B, Decramer M et al. Bronchodilator responsiveness in patients with COPD. Eur Respir J 2008; 31: 742 - 750

17 Mannino DM, Doherty DE, Sonia BA. Global Initiative on Obstructive Lung Disease (GOLD) classification of lung disease and mortality: findings from the Atherosclerosis Risk in Communities (ARIC) study. Respir Med 2006; 100: 115-122

18 Agusti A, Calverley PM, Celli B et al. Characterisation of COPD heterogeneity in the ECLIPSE cohort. Respir Res 2010; 11: 122

19 Spruit MA, Watkins ML, Edwards LD et al. Determinants of poor 6-min walking distance in patients with COPD: the ECLIPSE cohort. Respir Med 2010; 104: 849-857

20 Han MK, Muellerova H, Curran-Everett D et al. GOLD 2011 disease severity classification in COPDGene: a prospective cohort study. Lancet Respir Med 2013; 1: 43-50

21 Jones PW, Harding G, Berry P et al. Development and first validation of the COPD Assessment Test. Eur Respir J 2009; 34: 648-654

22 Jones PW, Harding G, Wiklund I et al. Tests of the responsiveness of the COPD assessment test following acute exacerbation and pulmonary rehabilitation. Chest 2012; 142: $134-140$

23 Mackay AJ, Donaldson GC, Patel AR et al. Usefulness of the Chronic Obstructive Pulmonary Disease Assessment Test to evaluate severity of COPD exacerbations. Am J Respir Crit Care Med 2012; 185: 1218 1224

24 van der Molen T, Willemse BW, Schokker S et al. Development, validity and responsiveness of the Clinical COPD Questionnaire. Health Qual Life Outcomes 2003; 1: 13

25 Lange P, Marott JL, Vestbo J et al. Prediction of the clinical course of chronic obstructive pulmonary disease, using the new GOLD classification: a study of the general population. Am J Respir Crit Care Med 2012: 186: $975-981$

26 Barnes PJ. Chronic obstructive pulmonary disease. N Engl J Med 2000; 343: $269-280$

27 Watz H, Waschki B, Boehme C et al. Extrapulmonary effects of chronic obstructive pulmonary disease on physical activity: a cross-sectional study. Am J Respir Crit Care Med 2008; 177: 743-751

28 Eickhoff P, Valipour A, Kiss D et al. Determinants of systemic vascular function in patients with stable chronic obstructive pulmonary disease. Am J Respir Crit Care Med 2008; 178: 1211-1218

29 Sin DD, Anthonisen NR, Soriano JB et al. Mortality in COPD: Role of comorbidities. Eur Respir J 2006; 28: 1245-1257

30 Agusti AG, Thomas A. Neff Lecture. Chronic Obstructive Pulmonary Disease: A Systemic Disease. Proc Am Thorac Soc 2006; 3: 478 - 481

31 Huertas A, Testa $U$, Riccioni R et al. Bone marrow-derived progenitors are greatly reduced in patients with severe COPD and low-BMI. Respir Physiol Neurobiol 2010; 170: 23-31

32 Huertas A, Palange $P$. Circulating endothelial progenitor cells and chronic pulmonary diseases. Eur Respir J 2011; 37: 426-431

33 Erb-Downward JR, Thompson DL, Han MK et al. Analysis of the lung microbiome in the "healthy" smoker and in COPD. PLoS One 2011; 6: e16384
34 Jiang $W$, Lederman $M M$, Hunt $P$ et al. Plasma levels of bacterial DNA correlate with immune activation and the magnitude of immune restoration in persons with antiretroviral-treated HIV infection. J Infect Dis 2009; 199: 1177-1185

35 Engelmann B, Massberg S. Thrombosis as an intravascular effector of innate immunity. Nat Rev Immunol 2013; 13: 34-45

36 Barr RG, Bluemke DA, Ahmed FS et al. Percent emphysema, airflow obstruction, and impaired left ventricular filling. N Engl J Med 2010; 362: $217-227$

37 Garcia-Aymerich J, Gomez FP, Benet $M$ et al. Identification and prospective validation of clinically relevant chronic obstructive pulmonary disease (COPD) subtypes. Thorax 2011; 66: 430-437

38 Holguin F, Folch E, Redd SC et al. Comorbidity and mortality in COPDrelated hospitalizations in the United States, 1979 to 2001. Chest 2005; 128: 2005-2011

39 Feary JR, Rodrigues LC, Smith CJ et al. Prevalence of major comorbidities in subjects with COPD and incidence of myocardial infarction and stroke: a comprehensive analysis using data from primary care. Thorax 2010; 65: 956-962

40 Donaldson GC, Hurst JR, Smith CJ et al. Increased risk of myocardial infarction and stroke following exacerbation of COPD. Chest 2010; 137: $1091-1097$

41 Maclay JD, McAllister DA, Johnston S et al. Increased platelet activation in patients with stable and acute exacerbation of COPD. Thorax 2011; 66: 769- 774

42 Iwamoto $H$, Yokoyama A, Kitahara $Y$ et al. Airflow limitation in smokers is associated with subclinical atherosclerosis. Am J Respir Crit Care Med 2009; 179: 35-40

43 Mascarenhas J, Lourenco P, Lopes $R$ et al. Chronic obstructive pulmonary disease in heart failure. Prevalence, therapeutic and prognostic implications. Am Heart J 2008; 155: 521 - 525

44 De Blois J, Simard S, Atar D et al. COPD predicts mortality in HF: the Norwegian Heart Failure Registry. J Card Fail 2010; 16: 225 - 229

45 Watz H, Waschki B, Meyer T et al. Decreasing cardiac chamber sizes and associated heart dysfunction in COPD: role of hyperinflation. Chest 2010; 138: $32-38$

46 Kessler R, Faller M, Weitzenblum E et al. "Natural history" of pulmonary hypertension in a series of 131 patients with chronic obstructive lung disease. Am J Respir Crit Care Med 2001; 164: 219-224

47 Arcasoy SM, Christie JD, Ferrari VA et al. Echocardiographic assessment of pulmonary hypertension in patients with advanced lung disease. Am J Respir Crit Care Med 2003; 167: 735 - 740

48 Chan AL, Juarez MM, Shelton DK et al. Novel computed tomographic chest metrics to detect pulmonary hypertension. BMC Med Imaging 2011; $11: 7$

49 Buch P, Friberg J, Scharling $H$ et al. Reduced lung function and risk of atrial fibrillation in the Copenhagen City Heart Study. Eur Respir J 2003; $21: 1012-1016$

50 Mannino DM, Thorn D, Swensen $A$ et al. Prevalence and outcomes of diabetes, hypertension and cardiovascular disease in COPD. Eur Respir J 2008; 32: $962-969$

51 Alexeeff SE, Litonjua AA, Sparrow $D$ et al. Statin use reduces decline in lung function: VA Normative Aging Study. Am J Respir Crit Care Med 2007; 176: $742-747$

52 Lahousse L, Loth DW, Joos GF et al. Statins, systemic inflammation and risk of death in COPD: the Rotterdam study. Pulm Pharmacol Ther 2013; 26: 212-217

53 Almagro P, Cabrera FJ, Diez J et al. Comorbidities and short-term prognosis in patients hospitalized for acute exacerbation of COPD: the EPOC en Servicios de medicina interna (ESMI) study. Chest 2012; 142: $1126-1133$

54 McAllister DA, Maclay JD, Mills NL et al. Diagnosis of myocardial infarction following hospitalisation for exacerbation of COPD. Eur Respir J 2012; 39: 1097-1103

55 Chang CL, Robinson SC, Mills GD et al. Biochemical markers of cardiac dysfunction predict mortality in acute exacerbations of COPD. Thorax 2011; 66: 764-768

56 Hoiseth $A D$, Neukamm A, Karlsson BD et al. Elevated high-sensitivity cardiac troponin $\mathrm{T}$ is associated with increased mortality after acute exacerbation of chronic obstructive pulmonary disease. Thorax 2011; 66: $775-781$

57 Soyseth $V$, Bhatnagar R, Holmedahl $\mathrm{NH}$ et al. Acute exacerbation of COPD is associated with fourfold elevation of cardiac troponin T. Heart 2013; 99: 122 - 126 
58 Simpson JA, Brunt KR, Collier CP et al. Hyperinflation-induced cardiorespiratory failure in rats. J Appl Physiol 2009; 107: 275-282

59 Wells JM, Washko GR, Han MK et al. Pulmonary arterial enlargement and acute exacerbations of COPD. N Engl J Med 2012; 367: 913-921

60 Duvoix A, Dickens J, Haq I et al. Blood fibrinogen as a biomarker of chronic obstructive pulmonary disease. Thorax 2012

61 Handschin C, Spiegelman BM. The role of exercise and PGC1alpha in inflammation and chronic disease. Nature 2008; 454: 463-469

62 Pedersen BK, Febbraio MA. Muscle as an endocrine organ: focus on muscle-derived interleukin-6. Physiol Rev 2008; 88: 1379-1406

63 Waschki B, Kirsten A, Holz $\mathrm{O}$ et al. Physical activity is the strongest predictor of all-cause mortality in patients with COPD: a prospective cohort study. Chest 2011; 140: 331-342

64 Suissa S, Assimes T, Ernst P. Inhaled short acting beta agonist use in COPD and the risk of acute myocardial infarction. Thorax 2003; 58 : 43-46

65 Salpeter SR, Ormiston TM, Salpeter EE. Cardiovascular effects of betaagonists in patients with asthma and COPD: a meta-analysis. Chest 2004; 125: 2309-2321

66 Campbell S, Criner G, Ziehmer B. Cardiac safety of formoterol in COPD [Poster P 1928]. Program and abstracts of the European Respiratory Society 15th Annual Congress;September 17-21, Copenhagen, Denmark: 2005

$67 \mathrm{Au} D \mathrm{DH}$, Udris EM, Fan VS et al. Risk of mortality and heart failure exacerbations associated with inhaled beta-adrenoceptor agonists among patients with known left ventricular systolic dysfunction. Chest 2003; 123: 1964-1969

68 Calverley PM, Anderson JA, Celli B et al. Salmeterol and fluticasone propionate and survival in chronic obstructive pulmonary disease. $\mathrm{N}$ Engl J Med 2007; 356: 775 - 789

69 Hilleman DE, Malesker MA, Morrow LE et al. A systematic review of the cardiovascular risk of inhaled anticholinergics in patients with COPD. Int J Chron Obstruct Pulmon Dis 2009; 4: 253-263

70 Anthonisen NR, Connett JE, Enright PL et al. Hospitalizations and mortality in the Lung Health Study. Am J Respir Crit Care Med 2002; 166 : $333-339$

71 Singh S, Loke YK, Furberg CD. Inhaled anticholinergics and risk of major adverse cardiovascular events in patients with chronic obstructive pulmonary disease: a systematic review and meta-analysis. JAMA 2008; 300: 1439-1450

72 Ogale SS, Lee TA, Au DH et al. Cardiovascular events associated with ipratropium bromide in COPD. Chest 2010; 137: 13-19

73 Celli B, Decramer M, Leimer I et al. Cardiovascular safety of tiotropium in patients with COPD. Chest 2010; 137: 20-30

74 Michele TM, Pinheiro S, Iyasu S. The safety of tiotropium - the FDA's conclusions. N Engl J Med 2010; 363: 1097-1099

75 Worth H, Chung KF, Felser JM et al. Cardio- and cerebrovascular safety of indacaterol vs formoterol, salmeterol, tiotropium and placebo in COPD. Respir Med 2011; 105: 571-579

76 Bateman E, Singh D, Smith $D$ et al. Efficacy and safety of tiotropium Respimat SMI in COPD in two 1-year randomized studies. Int J Chron Obstruct Pulmon Dis 2010; 5: 197-208

77 Wilchesky M, Ernst P, Brophy JM et al. Bronchodilator use and the risk of arrhythmia in COPD: part 1: Saskatchewan cohort study. Chest 2012; 142: 298 - 304

78 Wilchesky M, Ernst P, Brophy JM et al. Bronchodilator use and the risk of arrhythmia in COPD: part 2: reassessment in the larger Quebec cohort. Chest 2012; 142: $305-311$

79 Rabe KF. Drug safety in COPD revisited: what is the number needed to analyze? Chest 2012; 142: $271-274$

80 Shannon $M$. Life-threatening events after theophylline overdose: a 10-year prospective analysis. Arch Intern Med 1999; 159: 989-994

81 van der Hooft CS, Heeringa J, Brusselle GG et al. Corticosteroids and the risk of atrial fibrillation. Arch Intern Med 2006; 166: 1016-1020

82 White WB, Cooke GE, Kowey PR et al. Cardiovascular Safety In Patients Receiving Roflumilast for the Treatment of Chronic Obstructive Pulmonary Disease. Chest 2013
83 Swedberg K, Komajda M, Bohm M et al. Effects on outcomes of heart rate reduction by ivabradine in patients with congestive heart failure: is there an influence of beta-blocker dose? findings from the SHIFT (Systolic Heart failure treatment with the I(f) inhibitor ivabradine Trial) study. J Am Coll Cardiol 2012; 59: 1938 - 1945

84 Gottlieb SS, McCarter RJ, Vogel RA. Effect of beta-blockade on mortality among high-risk and low-risk patients after myocardial infarction. N Engl J Med 1998; 339: 489-497

85 Hawkins NM, Huang Z, Pieper KS et al. Chronic obstructive pulmonary disease is an independent predictor of death but not atherosclerotic events in patients with myocardial infarction: analysis of the Valsartan in Acute Myocardial Infarction Trial (VALIANT). Eur J Heart Fail 2009; 11: $292-298$

86 van Gestel YR, Hoeks SE, Sin DD et al. Impact of cardioselective betablockers on mortality in patients with chronic obstructive pulmonary disease and atherosclerosis. Am J Respir Crit Care Med 2008 178: $695-700$

$87 \mathrm{Au}$ DH, Bryson CL, Fan VS et al. Beta-blockers as single-agent therapy for hypertension and the risk of mortality among patients with chronic obstructive pulmonary disease. Am J Med 2004; 117: 925 931

88 Dransfield MT, Rowe SM, Johnson JE et al. Use of beta blockers and the risk of death in hospitalised patients with acute exacerbations of COPD. Thorax 2008; 63: $301-305$

89 Stefan MS, Rothberg MB, Priya A et al. Association between betablocker therapy and outcomes in patients hospitalised with acute exacerbations of chronic obstructive lung disease with underlying ischaemic heart disease, heart failure or hypertension. Thorax 2012; 67: 977-984

90 Short PM, Lipworth SI, Elder DH et al. Effect of beta blockers in treatment of chronic obstructive pulmonary disease: a retrospective cohort study. BMJ 2011; 342: d2549

91 Jensen MT, Marott JL, Lange P et al. Resting heart rate is a predictor of mortality in chronic obstructive pulmonary disease. Eur Respir $\mathrm{J} 2012$

92 Jorres $R A$, Welte T, Bals $R$ et al. [Systemic manifestations and comorbidities in patients with chronic obstructive pulmonary disease (COPD) and their effect on clinical state and course of the disease an overview of the cohort study COSYCONET]. Dtsch Med Wochenschr 2010; 135: 446- 449

93 Seeger $W$, Welte $T$, Eickelberg $O$ et al. [The German centre for lung research - translational research for the prevention, diagnosis and treatment of respiratory diseases]. Pneumologie 2012; 66: 464-469

94 Taube C, Kanniess F, Gronke L et al. Reproducibility of forced inspiratory and expiratory volumes after bronchodilation in patients with COPD or asthma. Respir Med 2003; 97: 568-577

95 Peters SP, Kunselman SJ, Icitovic $N$ et al. Tiotropium bromide step-up therapy for adults with uncontrolled asthma. N Engl J Med 2010; 363: $1715-1726$

96 Bateman ED, Kornmann O, Schmidt $P$ et al. Tiotropium is noninferior to salmeterol in maintaining improved lung function in B16-Arg/Arg patients with asthma. J Allergy Clin Immunol 2011; 128: 315-322

97 Kerstjens HA, Disse B, Schroder-Babo $W$ et al. Tiotropium improves lung function in patients with severe uncontrolled asthma: a randomized controlled trial. J Allergy Clin Immunol 2011; 128: 308 - 314

98 Kerstjens HA, Engel M, Dahl R et al. Tiotropium in asthma poorly controlled with standard combination therapy. N Engl J Med 2012; 367: $1198-1207$

99 Kummer W, Lips KS, Pfeil U. The epithelial cholinergic system of the airways. Histochem Cell Biol 2008; 130: 219-234

100 Raemdonck K, de Alba J, Birrell MA et al. A role for sensory nerves in the late asthmatic response. Thorax 2012; 67: 19-25

101 Bos IS, Gosens R, Zuidhof $A B$ et al. Inhibition of allergen-induced airway remodelling by tiotropium and budesonide: a comparison. Eur Respir J 2007; 30: 653-661

102 Kang JY, Rhee CK, Kim JS et al. Effect of tiotropium bromide on airway remodeling in a chronic asthma model. Ann Allergy Asthma Immunol 2012; 109: 29-35 